\title{
ON THE TIME SPENT ABOVE A LEVEL BY BROWNIAN MOTION WITH NEGATIVE DRIFT
}

\author{
J.-P. IMHOF, ${ }^{*}$ University of Geneva
}

\begin{abstract}
Limit theorems of Berman involve the total time spent by Brownian motion with negative drift above a fixed or exponentially distributed negative level. We give explicitly the probability densities and distribution functions, obtained via an equivalence of laws.
\end{abstract}

LAST PASSAGE: DURATION OF POSITIVITY

\section{Introduction}

If $B$ is standard Brownian motion, the total time $\xi$ that $2^{\frac{1}{2}} B(t)-t+Y$ spends above 0 , where $Y$ is a negative exponential variable with parameter 1 , independent of $B$, plays a substantial role in some limit theorems of Berman [1]. $\mathrm{He}$ has obtained the Laplace transform of $\xi$ and shown that if $\Gamma(t)=\operatorname{Pr}(\xi>t)$, then $-\Gamma^{\prime}(t)$ is a non-increasing function with $-\Gamma^{\prime}(0)=1$. Using an equivalence of laws which explains the squared form of Berman's result we obtain $\Gamma$ explicitly, identify $1+\Gamma^{\prime}$ as a distribution function and give also the density and distribution function when $y$ is constant.

\section{Results}

From now on, $\{X(t), t \geqq 0\}$ is the coordinate process on the space of continuous functions. We call $W_{-\delta}$ the law under which $X$ is Brownian motion with $X(0)=0$ and constant drift $-\delta$; only values $\delta \geqq 0$ are considered. Under $W=W_{0}, X$ is standard Brownian motion. Let $\tau(y)=\inf \{t>0: X(t)=y\}, M(t)=\max \{X(s): s \leqq t\}, \mu(t)=$ inf $\{s \leqq t: X(s)=M(t)\}$ and

$$
v(y ; t)=\int_{0}^{t} 1_{\{X(s)>y\}} d s .
$$

When $\delta>0, \mu=\mu(\infty)$ and $v(y)=v(y, \infty)$ are almost surely finite, and we write $v=v(0)$. We use $\tau(-Y)$ and $v(-Y)$ for random positive $Y$ also. Furthermore, $p(t ; x)=(2 \pi t)^{-\frac{1}{2}} \exp \left\{-x^{2} / 2 t\right\}, t>0, x \in \mathbb{R}$, and $\Phi$ is the standard normal distribution function.

Lemma 1. If $\delta>0$, the $W_{-\delta}$-laws of $\mu$ and $v$ are identical, with probability density

$$
2 \delta \psi(t ;-\delta)=2 \delta\left\{p(t ; \delta t)-\delta \Phi\left(-\delta t^{\frac{1}{2}}\right)\right\}, \quad t>0 .
$$

The Laplace transform is $L(\lambda ; \delta)=2 /\left\{1+\left(1+2 \lambda / \delta^{2}\right)^{\frac{1}{2}}\right\}, \lambda>0$.

Received 20 February 1986; revision received 17 July 1986.

* Postal address: Section de Mathématiques, Université de Genève 2-4, rue du Lièvre, Case Postale 240, 1211 Genève 24, Switzerland. 
Proof. First, let $\{X(t), 0 \leqq t \leqq s\}$ have the law of a Brownian bridge of duration $s$. Then $\mu(s)$ and $v(0, s)$ are well-known to be uniform over $[0, s]$. Considering the law of this Brownian bridge as weak limit, for $\varepsilon \downarrow 0$, of laws of Brownian motion conditioned to $X(s) \in[0, \varepsilon]$, one deduces the equality $W(\mu(s) \in d t, X(s) \in d 0)=W(v(0 ; s) \in d t, X(s) \epsilon$ $d 0), 0<t<s$. Referring to Example 6 of [2] this in turn implies, when $\delta>0$, $W_{-\delta}\left(\mu \in d t, \tau^{\prime}(0) \in d s\right)=W_{-\delta}\left(v \in d t, \tau^{\prime}(0) \in d s\right)$, where $\tau^{\prime}(0)=\sup \{t>0: X(t)=0\}$. Integration in $s$ gives $W_{-\delta}(v \in d t)=W_{-\delta}(\mu \in d t)=2 \delta \psi(t ;-\delta)$, the result for $\mu$ being known (see e.g. [2], Example 7). The Laplace transform is easily obtained.

Let $Y$ be a negative exponential variable with parameter $2 \delta$, independent of $X$ which has law $W_{-\delta}, \delta>0$. It is easy to see that when $\delta=2^{-\frac{1}{2}}$, the total time $v(-Y)$ during which $X(t)>-Y$ holds has the same law as Berman's $\xi$.

Lemma 2. Let $\delta>0$. The $W_{-\delta}$-law of $\tau(-Y)$ is the same as the law of $\mu$, and $v$, and the Laplace transform of $v(-Y)$ is $L^{2}(\lambda ; \delta), \lambda>0$.

Proof. For $y>0$, the $W_{-\delta}$-Laplace transform of $\tau(-y)$ is $\exp \left\{\delta y\left[1-\left(1+2 \lambda / \delta^{2}\right)^{\frac{1}{2}}\right]\right\}$ ([2], Lemma 3). That of $\tau(-Y)$ is therefore, at $\lambda>0$,

$$
2 \delta \int_{0}^{\infty} \exp (-2 \delta y) \exp \left\{\delta y\left[1-\left(1+2 \lambda / \delta^{2}\right)^{\frac{1}{2}}\right]\right\} d y=L(\lambda ; \delta) .
$$

Let $\theta$ be the shift operator. The total time $v(-y) \circ \theta(\tau(-y))$ spent by $X$ above $-y$, from $\tau(-y)$ on, is independent of $\tau(-y)$. As $Y$ is independent of $X, v(-Y) \circ \theta(\tau(-Y))$ and $\tau(-Y)$ are also independent. But $v(-Y)=\tau(-Y)+v(-Y) \circ \theta(\tau(-Y))$ where the second summand has, conditionally on $Y$ and therefore also unconditionally, the same law as $v$. The conclusion follows.

Once it is known that the $W_{-\delta}$-density of $v(-Y)$ is the convolution of $2 \delta \psi(t ;-\delta)$ with itself, it is only a matter of lengthy computation to obtain this density, and the corresponding distribution function. The same holds true for $v(-y)$, the density of $\tau(-y)$ being known (e.g. [2], 5.2). We record the results as follows.

Theorem. When $\delta>0, y>0$, one has for $t>0$ :

(a) $W_{-\delta}(\mu>t)=2 \Phi\left(-\delta t^{\frac{1}{2}}\right)-2 \delta t \psi(t ;-\delta)$.

(b) $W_{-\delta}(v(-Y) \in d t)=2 \delta^{2} W_{-\delta}(\mu>t) d t$.

(c) $W_{-\delta}(v(-Y)>t)=4 \Phi\left(-\delta t^{\frac{1}{2}}\right)-\left(1+\delta^{2} t\right) W_{-\delta}(\mu>t)$.

(d) $W_{-\delta}(v(-y) \in d t)=2 \delta \exp (2 \delta y)\left\{p(t ; y+\delta t)-\delta \Phi\left(-t^{\frac{1}{2}}(y+\delta t)\right)\right\} d t$.

(e) $W_{-\delta}(v(-y)>t)=\Phi\left(t^{-\frac{1}{2}}(y-\delta t)\right)+(1+2 \delta y) \exp (2 \delta y) \Phi\left(-t^{-\frac{1}{2}}(y+\delta t)\right)$ $-t W_{-\delta}(v(-y) \in d t) / d t$.

As pointed out before, (c) gives tor the particular value $\delta^{*}=1 / 2^{\frac{1}{2}}$, Berman's function $\Gamma(t)$, and (b) then shows that $-\Gamma^{\prime}(t)=W_{-\delta} \cdot(\mu>t)$.

\section{References}

[1] Berman, S. M. (1982) Sojourns and extremes of a diffusion process on a fixed interval. Adv. Appl. Prob. 14, 811-832.

[2] ImHOF, J. P. AND KÜMmerling, P. (1986) Operational derivation of some Brownian motion results. Int. Statist. Rev. 54, to appear. 